\title{
ESCLEROSE LATERAL AMIOTRÓFICA: UMA REVISÃO DO ESTADO DA ARTE PARA SISTEMAS DE MONITORAMENTO EM HOMECARE
}

\author{
Antônio Higor Freire de Morais \\ Mestre em Engenharia de Computação, Professor do Instituto Federal de Educação, \\ Ciência e Tecnologia do Rio Grande do Norte, atuando nas áreas de Sistemas Embarcados \\ Tolerantes a Falha e Automação Hospitalar. \\ E-mail: higor.morais@ifrn.edu.br \\ Ricardo Alexandro de Medeiros Valentim \\ Doutor em Engenharia Elétrica e de Computação, Coordenador do Laboratório de \\ Inovação Tecnológica em Saúde do Hospital Universitário Onofre Lopes, Professor do \\ Departamento de Engenharia Biomédica da Universidade Federal do Rio Grande do Norte. \\ E-mail: ricardo.valentim@ufrnet.br \\ Gláucio Bezerra Brandão \\ Professor da Universidade Federal do Rio Grande do Norte (UFRN) \\ E-mail: glaucio@dca.ufrn.br
}

\section{RESUMO}

Esse artigo apresenta uma revisão bibliográfica do Estado da Arte dos trabalhos publicados nas bases de pesquisa do IEEE, Scielo, Elsevier e CAPES, nos últimos 2 anos, referentes aos sistemas de monitoramento homecare para Esclerose Lateral Amiotrófica (ELA). Foram encontrados 2 artigos que tratam especificamente de sistemas de monitoramento em homecare para ELA e outros 9 artigos que não tratam especificamente, mas que podem ser adaptados para tal. Diante das características dos diversos sistemas, os trabalhos apresentados oferecem diversas funções que favorecem o atendimento/cuidado de pacientes em casa, bem como a melhoria da qualidade de vida.

PALAVRAS-CHAVE: Esclerose Lateral Amiotrófica (ELA), Sistema de Monitoramento Homecare, Estado da Arte.

\section{Amyotrophic Lateral Sclerosis: The state of art review for homecare monitoring systems}

\begin{abstract}
This paper presents a survey of state of art in Works published on research bases of IEEE, Scielo, Elsevier and Capes in last 2 years related to homecare monitoring systems for Amyotrophic Lateral Sclerosis (ALS). It were found 2 papers specific related to homecare monitoring system to ALS and others 9 papers that are not specific related to but which can be adapted. Despite of such system characteristics, the presented works offers many functions which favors the attend/care of patients in home, as well as the quality of life improvement.
\end{abstract}

KEY-WORDS: Amyotrophic Lateral Sclerosis (ALS), Homecare Monitoring System, State of Art 


\section{ESCLEROSE LATERAL AMIOTRÓFICA: UMA REVISÃO DO ESTADO DA ARTE PARA SISTEMAS DE MONITORAMENTO EM HOMECARE}

\section{INTRODUÇÃO}

A Qualidade de Vida (QV) é uma medida que está diretamente ligada ao senso comum e de complexa quantificação. Esta complexidade de mensuração deve-se essencialmente a fatores subjetivos associados a cada indivíduo. Neste sentido, não existe na literatura um consenso a cerca do tema, mas acredita-se que alguns elementos como: experiência de vida, quais sejam, valores humanos e culturais; a multidimensionalidade do ser, que está diretamente relacionada ao ambiente em que este vive são preponderantes para determinação desta qualidade de vida [1]. Nos ambientes hospitalares, essa medida requer que seus parâmetros sejam avaliados de forma rígida, haja vista as condições exaustivas e adversas enfrentadas pelos pacientes, familiares e equipe de saúde. Nesse caso, segundo Coco et. al. [2], embora não haja correlação entre a qualidade de vida da equipe de saúde e os pacientes, ambos apresentaram baixos índices em relação a este quesito. Sendo assim, a atenção que deve ser dispensada aos pacientes em situações de risco acaba por exigir ainda mais da equipe de saúde envolvida. Desta forma, a utilização de sistemas que permitam melhorar a eficiência nos tratos aos pacientes, por meio de sistemas de monitoramento vem se tornando uma praxe nos hospitais [3].

As vantagens advindas do uso desse sistema são muitas, pois essa tecnologia exerce sua tarefa de forma ininterrupta, quando necessário, e com extrema precisão e exatidão. Dessa forma, a possibilidade de erros em decorrência de fadiga ou cansaço é minimizada, haja vista que os sistemas de monitoramento não estão sujeitos a erros decorrentes do desgaste físico e/ou mental e podem emitir alertas quando situações de risco são detectadas. Além disso, os sistemas são capazes de apresentar respostas a uma situação de risco em tempo muito menor, devido a sua capacidade de processamento, monitoração constante e interligação com diversos outros sistemas. Neste caso, vários membros de uma equipe de saúde podem ser informados ao mesmo tempo, por meio de mensagens, sobre a ocorrência de uma situação de risco com o paciente. É possível também, direcionar de forma mais precisa o envio dessas mensagens, acionando os membros da equipe de acordo com o tipo de situação em que o paciente esteja envolvido. Em certos casos, somente uma parte da equipe de saúde deve ser notificada, dessa forma, evita-se o envio excessivo das mensagens de alertas. Além disso, a seleção dessas mensagens é de fundamental importância para que os alertas não se tornem banalizados pela própria equipe de saúde. Não obstante, um sistema de monitoramento também é capaz de realizar a verificação de várias informações sobre o estado do paciente (taxa de oxigenação, taxa de glicose, frequência cardíaca, frequência respiratória) ao mesmo tempo, processá-las e obter uma resposta inicial de acordo com o algoritmo de análise dessas informações.

A Esclerose Lateral Amiotrófica (ELA) é uma doença neurodegenerativa, caracterizada por fraqueza muscular progressiva que leva o paciente ao óbito, usualmente devido a complicações respiratórias. Todavia, a fisioterapia, por meio de intervenções cinesioterapêuticas e orientações aos pacientes e aos cuidadores, é capaz de amenizar a perda físico-funcional, a fadiga muscular e a imobilidade do sistema músculo-esquelético, melhorando a qualidade de sobrevida [4]. A degeneração dos neurônios motores inferiores e superiores que atinge a medula espinhal, o tronco encefálico e o córtex motor resultam na definição da ELA como doença do neurônio motor. No momento que os músculos ventilatórios são comprometidos os indivíduos apresentam restrições pulmonares, 
caracterizadas por redução da Capacidade Vital (CV) e do Volume Corrente (VC) com, consequente, insuficiência respiratória crônica. A Ventilação Não-Invasiva (VNI) consta na administração de ventilação mecânica aos pulmões sem que haja a necessidade de vias aéreas artificiais, podendo ser oferecida ao paciente por meio de ventiladores mecânicos ou através de aparelhos denominados Pressão Positiva Bifásica nas Vias Aéreas (Bilevel).

Quando estes pacientes estão internados em ambiente hospitalar, a perda de calibração dos instrumentos e dispositivos que são utilizados no auxilio à respiração dos pacientes pode ser facilmente corrigida pelo técnico especialista da área de saúde que esteja responsável. No entanto, quando se trata de um paciente que está recebendo cuidados em casa, o contexto é outro, pois nem sempre é possível ter um técnico especialista que possa garantir que o equipamento estará sempre em sincronia com as necessidades do paciente com ELA. Essa tarefa é realizada nestes casos por um familiar ou um cuidador. Em se tratando de pacientes do Sistema Único de Saúde (SUS), a realidade é ainda mais grave, pois em geral esses familiares são mais carentes e em muitos casos não podem pagar por um cuidador capacitado que possa atender a esse paciente. Neste caso, o atendimento que é prestado ao paciente é realizado por um familiar que não possui o mesmo preparo que um especialista. Dessa forma, mesmo com a disponibilização do aparelho de ventilação não-invasiva fornecido pelo SUS, a utilização deste pode acontecer de forma ineficiente. Porém, a constituição brasileira assegura que todo cidadão brasileiro tem direito a assistência médica independente de sua condição financeira.

As sessões a seguir estão divididas na seguinte forma: a sessão 2 apresenta os trabalhos relacionados à sistemas homecare para ELA, além de outros gerais; a sessão 3 apresenta os resultados que podem ser depreendidos desses trabalhos; a sessão 4 apresenta uma discussão a cerca desses trabalhos; e a sessão 5, tendo em vista que nenhum trabalho é conclusivo e finalizado nele próprio, apresenta sugestões de trabalhos que podem ser desenvolvidos de acordo com as lacunas deixados pelos autores.

\section{SISTEMAS DE MONITORAMENTO}

Os sistemas de monitoramento, de uma forma geral, são utilizados para realizar tarefas que são demasiadamente desgastantes para os homens e que necessitam de uma atenção constante para um determinado processo. Tais sistemas são constituídos de componentes eletrônicos com poder de processamento, além de sensores e atuadores, e, em alguns casos, elementos mecânicos ou eletromecânicos. Pelo fato de não sofrem desgastes físicos decorrentes de cansaço ou fadiga, eles são capazes de atuar de forma ininterrupta sobre um determinado processo. Além disso, são capazes de realizar o monitoramento de muitas variáveis ao mesmo tempo e gerar uma resposta em um tempo mais rápido, quando comparado a observadores humanos. $\mathrm{Na}$ indústria, estes sistemas são chamados de Sistemas Supervisórios e estão amplamente difundidos, mas, em ambientes hospitalares, tais sistemas ainda alcançaram o mesmo nível de maturidade, pois os aspectos observados no desenvolvimento desses sistemas para a indústria são diferentes da área da saúde [5]. Porém, cabe ressaltar que os aspectos observados para o desenvolvimento de sistemas industriais são diferentes dos adotados para ambientes hospitalares ou domiciliares (homecare). A indústria petroquímica, por exemplo, dispõe de várias áreas que estão sujeitas à ocorrência de incêndios e explosões. Estas áreas são chamadas de áreas classificadas e, neste caso, os equipamentos que são instalados nestas áreas necessitam de um grau de segurança e proteção diferenciado. Porém esta mesma situação não acontece maior parte dos setores hospitalares ou domicílios, salvo ambientes específicos, como é o 
caso de cozinhas. Ainda assim, o risco de incêndio ainda é muito menor quando comparado às áreas classificadas da indústria.

\subsection{Sistema de Monitoramento Homecare para ELA}

A ELA é uma doença para a qual muitas pesquisas ainda estão sendo realizadas a fim de descobrir a real causa da mesma [6]. Por essa mesma razão foram encontradas poucas referências de sistemas de monitoramento desenvolvidos especificamente para $\mathrm{o}$ acompanhamento desses pacientes, as quais serão descritas a seguir.

Bustamante et. al. [12] apresentam o desenvolvimento de uma arquitetura de um sistema para monitoramento de pacientes que são acometidos pelas Doença de Parkinson (DP) e ELA baseado na realização de testes para verificar o grau de evolução das doenças. A arquitetura consiste de uma luva dotada de sensores para realização de dois testes: o teste de bater com os dedos continuamente (Finger Tapping Test) e o teste de força quando o paciente agarra um objeto (Hand-grip Strength Test); um dispositivo microprocessado acoplado à luva e recebe os sinais enviados pelos sensores da mesma e armazena essas informações em uma unidade de memória; por fim, uma aplicação de software para PC que recebe as informações armazenados no dispositivo microprocessado, através de uma interface USB, e as disponibiliza por meio de gráficos para a equipe de saúde.

Madani et. al. [8] e Baga et. al. [9] sugerem a criação de um sistema de monitoramento e possível modelagem do comportamento de pacientes afetados por DP e ELA. Para tanto, o sistema é capaz de gerenciar e monitorar o paciente em domicílio, por meio de identificação de status do paciente (on/off), tremores, discinesia induzida por medicamentos e eventos de queda. O sistema como um todo pode ser entendido em três partes: um conjunto de dispositivos que constituem uma rede de sensores que devem ser colocados no paciente, ou constituírem parte de uma peça do vestuário como uma camisa ou luva, tais como acelerômetro, giroscópio, electrooculograph ${ }^{1}$ (EOG), oxímetro de pulso e sensor de pele galvânico; outro elemento que também é parte desse sistema é uma unidade de processamento local que coleta todos os dados gerados pela rede de sensores e envia-os para a unidade central do hospital, além disso, realiza uma série de outras atividades tais como: verificação da sincronização e coordenação dos sensores, identificação das tarefas agendadas realizadas e não realizadas, geração de alertas para o paciente; a unidade central do hospital proporciona uma interface para a equipe de saúde por analisar os dados do paciente, bem como prescrever testes para realizados e/ou medicamentos ingeridos.

\subsection{Sistema de Monitoramento Homecare}

Como não foram encontradas muitas referências para sistemas de monitoramento homecare para ELA, resolvemos apresentar os trabalhos mais recentes que tratam do tema de forma mais abrangente. Alguns desses trabalhos sugerem a criação de modelos arquiteturais para desenvolvimento de sistemas, enquanto outros apresentam um sistema em si.

\footnotetext{
1 Trata-se de um dispositivo constituído por dois eletrodos que são fixados ao lado dos olhos e é capaz de detectar o movimento desses por meio da medição do diferencial de voltagem que é gerado por este movimento quando o indivíduo olha para esquerda ou para a direita.
} 
Em se tratando de ambientes domiciliares, a computação ubíqua (ou onipresente) apresenta-se como uma alternativa interessante para o desenvolvimento de sistemas de monitoramento de pacientes, pois estes pacientes, apesar de não apresentarem um quadro clínico grave, necessitam de cuidados e atenção que podem acontecer de forma ininterrupta através desses sistemas. Neste sentido, Carvalho et. al. [10], propõem a concepção de um sistema computacional inteligente para interligar o paciente em sua casa e os profissionais de saúde, por meio da integração de diversos serviços relacionados ao monitoramento remoto da saúde do paciente. Para tanto, os autores sugerem o desenvolvimento de um elemento central de processamento de dados fisiológicos do paciente e variáveis do ambiente domiciliar, como umidade do ar e temperatura, por meio de sensores instalados na casa do paciente que enviam dados para uma central de supervisão médica. Esse sistema é capaz de receber instruções de prescrições médicas que devem ser seguidas pelo paciente e, este por sua vez, recebe estas informações e alertas através de dispositivos de comunicação móvel (tablet, smartphones, etc.) ou outros aparelhos com capacidade de conexão com rede e processamento.

O InteliMed [11] é um sistema móvel de suporte à decisão de apoio ao diagnóstico médico utilizando árvores de decisão. Tal sistema é constituído por três módulos: Aplicação Móvel; Servidor Intermediário; e Servidor Inteligente. O módulo de Aplicação Móvel é o responsável por permitir operações de campo, incluindo cadastro de pacientes, diagnósticos e visualização das visitas por funcionário. O Servidor Intermediário é a interface entre a Aplicação Móvel e o Servidor Inteligente, possuindo um banco de dados local e permitindo a sincronização de dados entre ambos. O Servidor Inteligente executa a técnica da construção e modelagem de árvore de decisão sobre uma base de dados consolidada de pacientes em uma determinada especialidade médica. Esse sistema apresenta ainda as seguintes características: arquitetura cliente-servidor baseada no modelo de filas; processamento local do cliente o que permite uma menor dependência do servidor e um melhor balanceamento de carga; conexão assíncrona com os servidores, somente no momento de autenticação e encerramento da conexão; e transmissão de dados do tipo armazena e envia (store-and-forward).

Um exemplo claro de trabalho que apresenta uma visão conceitual por meio de ontologias para o desenvolvimento de sistemas pervasivos é o descrito por Freitas et. al. [12]. A modelagem arquitetural apresentada sugere o desenvolvimento de sistemas baseados em um conjunto de hardware e sensores que devem ser instalados no domicílio do paciente e um sistema de armazenamento em nuvem que irá receber os dados desse. Dessa forma, a equipe de saúde envolvida no processo avalia os dados dos pacientes que estão armazenados na nuvem. Esse conjunto de ontologias tem por objetivo facilitar o desenvolvimento de sistemas voltados para homecare, porém aspectos relacionados à segurança, dependabilidade e privacidade dos dados não estão contemplados.

A arquitetura proposta por Ha \& Byun [13] trata-se de um sistema composto por um servidor de controle doméstico, dispositivos que devem ser usados juntamente com peças de roupas, uma rede de sensores Zigbee e um provedor de serviços para cuidados da saúde do paciente. O diferencial desse trabalho é a utilização do framework Open Services Gateway initiative (OSGi) que funciona sobre plataforma Java e oferece o suporte necessário para o desenvolvimento da interface e integração do sistema com os dispositivos de hardware. Sendo assim, todo o cerne do controle homecare é desenvolvido sobre esse framework. 
Ribeiro et. al. [14] desenvolveram um sistema de supervisão homecare que monitora a frequência cardíaca do paciente, pressão sanguínea e temperatura corporal por meio de sensores, colocados no paciente, e que se comuniquem com uma aplicação para smartphone desenvolvida em J2ME. A ideia central do sistema consiste em permitir que o mesmo possa se comunicar com uma unidade central por meio de redes ethernet (802.3) ou Wi-Fi (802.11) quando o paciente se encontra no hospital e através de GPRS (General Packet Radio Service) quando estiverem fora. O sistema conta ainda com um Servidor Web que armazena os dados do paciente que serão analisados pela equipe de saúde.

Outro foco de desenvolvimento de trabalhos que está sendo abordado diz respeito à utilização de smartphones no auxilio a criação de sistemas homecare. Nesse contexto, Kaneyasu \& Akiyama [15] realizaram um trabalho focado na avaliação da segurança da informação no que se refere à privacidade dos dados dos pacientes baseado na autenticação de pacientes através de cartões SIM (Subscriber Identity Module). O procedimento para acessar o sistema consiste em três etapas: conectar-se a uma rede VPN (Virtual Private Network); autenticação com o cartão SIM; e autenticação por meio de ID própria do usuário. As informações são passadas para o sistema pelo cuidador ou pelo próprio paciente e, depois de enviadas para um servidor central, elas são apagadas do smartphone como forma de aumentar o grau de proteção do sistema em caso de perda ou extravio do aparelho.

Adotando também a linha da portabilidade e funcionalidade dos smartphones, Lee [16] apresenta o desenvolvimento de um sistema que auxilia o acompanhamento médico por meio de preenchimento digital do formulário de dados do paciente em uma aplicação para smartphone. Esse preenchimento acontece quando os pacientes recebem a visita em casa de uma equipe de saúde. Além do preenchimento de dados de texto sobre a situação do paciente, imagens também podem ser armazenadas no sistema.

Zhou et. al. [17] desenvolveram um sistema de tomada de decisão baseado em ambiente inteligente sensível ao contexto chamado EHA (Elderly in-home assistance). O trabalho é direcionado a cuidados a idosos tendo como base o conhecimento prévio de algumas atividades que estes podem realizar em um determinado ambiente, tais como tomar uma medicação, assistir TV, escovar os dentes, lavar o rosto ou vestir uma roupa. Dessa forma, o sistema é capaz de identificar, prever, interpretar e atuar de acordo com a atividade diária que o idoso esteja realizando e assisti-lo, como por exemplo, lembrando-o de tomar determinado medicamento ou avisando-o que ainda não é o momento para tal ação. Tratase de um projeto conceitual que foi validado segundo um conjunto de variáveis que descrevem as informações de contexto em um caso particular de EHA, para o qual foram definidas 20 situações possíveis de tarefas.

Seguindo a mesma linha de trabalho voltado para cuidado de pessoas idosas, Naeemabadi et. al. [18] apresentam uma plataforma de comunicação confiável para sistemas de homecare. Neste caso, os autores fazem uma análise de uma série de tipos de comunicação que podem ser utilizados para longas e curtas distâncias, bem como uma série de dispositivos para essa transmissão. A comunicação a curta distância é utilizada para o envio de dados entre uma Unidade Móvel responsável pela captura dos sinais vitais analisados e a Unidade Central Doméstica (UCD), que realiza o controle e avaliação desses dados, além de enviá-los para a Unidade de TeleCare (UTC). A comunicação de longa distância é feita entre a UCD e UTC. Após uma série de análises os autores sugerem os melhores padrões de comunicação e dispositivos, dentre os analisados. 


\section{RESULTADOS}

A pesquisa de revisão dos trabalhos desenvolvidos concentrou-se em trabalhos publicados a partir do ano de 2010 e em bases de pesquisas do IEEExplorer, periódicos da CAPES, SCIELO, Elsevier e referências do Google Acadêmico, neste último caso, sendo verificada a veracidade da publicação. No total, foram avaliados 12 artigos, porém dois artigos [8] e [9] referem-se a um mesmo trabalho, apesar de se tratarem de autores diferentes, totalizando um total de 11 trabalhos avaliados. $\mathrm{Na}$ base de dados da Elsevier não encontramos artigos que tratavam especificamente de sistemas de monitoramento em homecare para ELA, nem de monitoramento em homecare de forma genérica.

Como forma de catalogar as diversas características dos vários sistemas propostos, resolvemos construir uma tabela para melhorar a compreensão global dos trabalhos e facilitar a análise e comparação entre os mesmos. Nesse sentido, a Tabela XX reúne esses dados para que possamos analisá-los.

\begin{tabular}{|c|c|c|c|c|c|c|c|c|c|c|c|}
\hline Artigo & CPH & CPD & SP & GG & RSSV & RSA & $\begin{array}{l}\text { ALE } \\
\text { Ou } \\
\text { PRE }\end{array}$ & ARM & DM & $\begin{array}{l}\text { AUT } \\
\text { ou } \\
\text { SEG }\end{array}$ & PC \\
\hline$[7]$ & $\mathrm{X}$ & $\mathrm{X}$ & $\mathrm{X}$ & $\mathrm{X}$ & - & - & - & - & - & - & - \\
\hline$[8][9]$ & $\mathrm{X}$ & $\mathrm{X}$ & $\mathrm{X}$ & - & $\mathrm{X}$ & - & $\mathrm{X}$ & - & - & - & - \\
\hline$[10]$ & $\mathrm{X}$ & $\mathrm{X}$ & - & - & $\mathrm{X}$ & $\mathrm{X}$ & $\mathrm{X}$ & $\mathrm{X}$ & $\mathrm{X}$ & - & - \\
\hline$[11]$ & $\mathrm{X}$ & $\mathrm{X}$ & - & - & - & - & - & $\mathrm{X}$ & $\mathrm{X}$ & $\mathrm{X}$ & - \\
\hline$[12]$ & $\mathrm{X}$ & $\mathrm{X}$ & $\mathrm{X}$ & - & - & $\mathrm{X}$ & - & - & - & - & $\mathrm{X}$ \\
\hline$[13]$ & - & $\mathrm{X}$ & $\mathrm{X}$ & - & - & - & - & - & - & - & - \\
\hline$[14]$ & $\mathrm{X}$ & $\mathrm{X}$ & - & - & $\mathrm{X}$ & - & - & - & $\mathrm{X}$ & - & - \\
\hline$[15]$ & $\mathrm{X}$ & - & - & - & - & - & - & - & $\mathrm{X}$ & $\mathrm{X}$ & - \\
\hline$[16]$ & - & - & - & - & - & - & $\mathrm{X}$ & - & $\mathrm{X}$ & - & - \\
\hline$[17]$ & - & - & - & - & - & $\mathrm{X}$ & $\mathrm{X}$ & - & - & - & $\mathrm{X}$ \\
\hline$[18]$ & $\mathrm{X}$ & $\mathrm{X}$ & - & - & - & $\mathrm{X}$ & - & - & - & - & - \\
\hline
\end{tabular}

CPH: Central de Processamento Hospitalar CPD: Central de Processamento Domiciliar SP: Sensores pervasivos

GG: Geração de Gráficos

RSSV: Rede de Sensores de Sinais Vitais

RSA: Rede de Sensores do Ambiente
ALE ou PRE: Alertas ou Prescrição

ARM: Armazenamento de dados (servidor)

DM: Dispositivos Móveis

AUT ou SEG: Autenticação ou Segurança

PC: Projeto Conceitual ou Arquitetural

\section{DISCUSSÃO}

Nos hospitais, grande parte dos sistemas de monitoramento de pacientes faz uso de monitores multiparamétricos que apresentam algumas limitações no que diz respeito ao envio/disponibilização desses sinais vitais e integração com outros sistemas. Em se tratando de sistemas de monitoramento em homecare, percebemos que uma das principais preocupações dos autores é permitir que os dados colhidos no ambiente domiciliar sejam enviados para alguma central médica. Dentre os 12 trabalhos analisados, 8 apresentaram essa preocupação. Isso é importante, pois permite que a equipe de saúde possa desenvolver suas atividades de forma mais eficiente eliminando visitas desnecessárias tanto por parte 
dela, quanto do paciente ao hospital. Dentre todas as características analisadas nos diversos sistemas de monitoramento homecare, a utilização de um sistema de processamento de dados distribuído constituiu-se como um fator evidente em quase todos os trabalhos.

Outro fator interessante diz respeito aos sensores pervasivos, que são sensores que são utilizados juntamente com alguma peça de roupa, como uma luva ou camisa, que permite a realização de algumas atividades de testes fisioterapêuticos, principalmente para os casos de ELA. Porém, analisando o cenário dos diversos sistemas de monitoramento homecare essa característica dos demais sistemas não é algo recorrente, estando presente em apenas outros dois trabalhos.

No que diz respeito à utilização de dispositivos móveis para auxílio ou suporte desses sistemas, 5 trabalhos fazem uso desses dispositivos. Esses dispositivos, notadamente os smartphones, estão cada vez mais presentes em nosso cotidiano. Associando esse fator a sua capacidade de integração com outras tecnologias, além do seu poder de processamento, demonstra que a utilização desses aparelhos apresenta-se como um componente interessante que pode ser acoplado aos diversos sistemas de monitoramento homecare. Nos trabalhos analisados sua função principal diz respeito à parte gerencial de algumas atividades que devem ser realizadas pelos pacientes. Nesse caso, os smartphones podem lembrar o paciente sobre a execução de alguma atividade (tomar medicamento, por exemplo) por meio de geração de alertas ou receber alguma prescrição médica.

Por se tratar de monitoramento em homecare, o ambiente domiciliar tem impacto direto sobre as ações de monitoramento do paciente pelo sistema. Sendo assim, alguns trabalhos consideraram importante a medição de algumas variáveis desses ambientes, como temperatura e umidade, além também de identificarem a presença do paciente nos diversos ambientes da casa. Essa prática é mais comum em sistemas de monitoramento homecare para idosos, devido a maior susceptibilidade a quedas, mas tais aplicações podem ser aplicadas a pacientes com dificuldades motoras de locomoção.

No que se refere aos critérios de segurança e dependabilidade do sistema, bem como a proteção e sigilo dos dados dos pacientes, poucos trabalhos mostraram-se preocupados quanto a esse quesito. Não obstante, não foram encontrados trabalhos que apresentassem prognósticos do quadro evolutivo dos pacientes nem foram específicos quando da possibilidade de comparação de dados em momentos distintos.

\section{CONCLUSÃO}

Os trabalhos apresentados oferecem diversas características que favorecem $o$ atendimento/cuidado de pacientes em casa, bem como a melhoria da qualidade de vida. Diante dos aspectos expostos nas sessões de Resultados e Discussão percebemos que existem poucos trabalhos de homecare voltados exclusivamente para o tratamento de pacientes com essa doença. Isso demonstra que esse é um nicho de pesquisa científico pouco explorado e que pode ser objeto de estudo de outros pesquisadores. Além disso, é preciso observar que os sistemas de monitoramento em homecare dos trabalhos apresentados não mostraram informações importantes no tocante à confiabilidade, integridade dos dados, dois requisitos importantes que podem influenciar diretamente no diagnóstico e perspectivas de melhorias do quadro do paciente. Dessa forma, podemos perceber que trabalhos que abordem essa linha de pesquisa ainda podem ser desenvolvidos, avaliando principalmente os requisitos de sincronização de dados dos sistemas. 


\section{REFERÊNCIAS BIBLIOGRÁFICAS}

1. ORSINI, M. MELLO, M., LISIEUX, D., PÁSSARO, C.P., LEITE, M.A.A., BALDEZ, A.C., SILVA, J.G., MENEZES, S.L.S., PORTO, F.H., MACHADO, D., BASTO, V.H., FREITAS, M.R.G., OLIVEIRA, A.B. "Qualidade de Vida de Cuidadores e Pacientes com Diagnóstico de Esclerose Lateral Amiotrófica", Revista de Neurociência, 2012.

2. COCO, G.L., COCO, D.L., CICERO, V., OLIVERI, A., VERSO, G.L., PICCOLI, F., BELLA, V.L. "Individual and health-related quality of life assessment in amyotrophic lateral sclerosis patients and their caregivers", Journal of the Neurological Sciences, V. 238, p. 11-17, 2005.

3. ARAÚJO, B., VALENTIM, R.A.M., LACERDA, J.M.T., CARVALHO, D.R., DANTAS, M.C.R., DINIZ JUNIOR, J. "Modelo arquitetural para geração de alertas aplicado ao monitoramento de pacientes em ambiente hospitalar", Revista Brasileira de Engenharia Biomédica, 2012.

4. POZZA, A.M., DELAMURA, M.K., RAMIREZ, C., VALÉRIO, N.I., MARINO, L.H.C., LAMARI, N.M. "Conduta Fisioterapêutica na esclerose lateral amiotrófica". Sao Paulo Medical Journal, v. 124, n. 6, p. 350-354, 2006. http://dx.doi.org/10.1590/S1516-31802006000600011

5. VALENTIM, R.A.M., ARAÚJO, B.G., LACERDA, J.M.T., SOUZA, P.C.O., CARVALHO, T.P.M. "Automação Hospitalar: O Estado da Arte", Revista Brasileira de Inovação Tecnológica em Saúde, v. 1, n. 1, p. 27-38, 2012.

6. ABRELA - Associação Brasileira de Esclerose Lateral Amiotrófica. "Manual sobre a Esclerose Lateral Amiotrófica, atualização 2010", Disponível em: <http://www.abrela.org.br/PDF/livreto_2010.pdf>, Acesso em: 29 março 2012.

7. BUSTAMANTE, P., GRANDEZ, K., SOLAS, G., ARRIZABALAGA, S. "A LowCost Platform for Testing Activities in Parkinson and ALS Patients", 12th IEEE International Conference on e-Health Networking Applications and Services (Healthcom), p. 302-307, 2010.

8. MADANI, K., LOHI, M., LOHI, A. "A Decision Support Service Platform for Neurodegenerative Disease Patients", 6th International Conference on Networking and Services (ICNS), p. 188-191, 2010 .

9. BAGA, D., FOTIADIS, D.I., KONITSIOTIS, S., TSOULI, S.; DIAKOU, M.; ARRENDONDO, M.T., ESTRADA, J.J., PANSERA, M., AKAY, M. "PERFORM: A platform for monitoring and management of chronic neurodegenerative diseases: The Parkinson and Amyotrophic Lateral Sclerosis case". 4th International IEEE/EMBS Conference on Neural Engineering, p. 1-5, 2009.

10. CARVALHO, S.T., COPETTI, A., LOQUES FILHO, O.G. "Sistema de computação ubíqua na assistência domiciliar à saúde", Journal Of Health Informatics, v. 3, n. 2, p. 51-57, 2011.

11. MENEZES JUNIOR, J.V., D'CASTRO, R.J., RODRIGUES, F.M.M., GUSMÃO, C.M.G., LYRA, N.R.S., SARINHO, S.W., "InteliMed: uma experiência de desenvolvimento de sistema móvel de suporte ao diagnóstico médico ", Revista Brasileira de Computação Aplicada, v. 3, n. 1, p. 30-42, 2011. 
12. FREITAS, L.O., LIBRELOTTO, G.R., PEREIRA, H.G.G., KASPER, J., MARTINI, R.G., MOZZAQUATRO, B., PEREIRA, R.T. "Applying pervasive computing in an architecture for homecare environments", 9th International Conference on Ubiquitous Intelligence and Computing, p. 185-192, 2012.

13. HA, Y. BYUN, Y. "A Ubiquitous Homecare Service System Using a Wearable User Interface Device", 11th International Conference on Computer and Information Science, p. 649-650, 2012.

14. RIBEIRO, A.G.C.D., MAITELLI, A.L., VALENTIM, R.A.M., BRANDÃO, G.B., GUERREIRO, A.M.G. "AngelCare Mobile System: Homecare patient monitoring using Bluetooth and GPRS", 32nd Annual International Conference of the IEEE EMBS, p. 2200-2203, 2010.

15. KANEYASU, F., AKIYAMA, M. "System Requirements for an Electronic Health Record System Using Smartphones for Homecare", Proceedings of PICMET '12: Technology Management for Emerging Technologies, p. 3059-3066, 2012.

16. LEE, C. "A Home Care Service Platform For Mobile Healthcare", Proceedings of the 2012 International Conference on Machine Learning and Cybernetics, p. 19271930, 2012.

17. ZHOU, F., JIAO, J., Chen, S., ZHANG, D. "A Case-Driven Ambient Intelligence System for Elderly in-Home Assistance Applications", IEEE Transactions On Systems, Man, And Cybernetics-Part C: Applications And Reviews, v. 41, n. 2, p.179-189, 2011.

18. NAEEMABADI, M., ZABIHI, M., ORDOUBADI, B.S., SALEH, M.A., KHALILZADEH, M.A., ORDOUBADI, M.S. "Tele-homecare System Design for elderly", 5th International Conference on Application of Information and Communication Technologies (AICT), p. 1-5, 2011. 